\title{
Le français de l'époque moderne comme objet d'histoire sociale et culturelle : un témoignage personnel
}

Willem Frijhoff

\section{(2) OpenEdition Journals}

Édition électronique

URL : https://journals.openedition.org/dhfles/3696

DOI : 10.4000/dhfles.3696

ISSN : 2221-4038

Éditeur

Société Internationale pour l'Histoire du Français Langue Étrangère ou Seconde

Édition imprimée

Date de publication : 1 janvier 2013

Pagination : 139-144

ISSN : 0992-7654

\section{Référence électronique}

Willem Frijhoff, «Le français de l'époque moderne comme objet d'histoire sociale et culturelle : un témoignage personnel », Documents pour l'histoire du français langue étrangère ou seconde [En ligne], 50 | 2013, mis en ligne le 01 janvier 2017, consulté le 26 mars 2023. URL : http:// journals.openedition.org/dhfles/3696; DOI : https://doi.org/10.4000/dhfles.3696

Ce document a été généré automatiquement le 26 mars 2023.

Tous droits réservés 


\title{
Le français de l'époque moderne comme objet d'histoire sociale et culturelle : un témoignage personnel
}

\author{
Willem Frijhoff
}

1 Depuis bientôt un quart de siècle l'histoire du français en tant que langue étrangère ou seconde constitue une part importante de ma recherche d'historien de la culture, de l'éducation et des sociétés. Je ne suis ni linguiste ou littérateur, ni pédagogue ou éducateur au sens strict, universitaire ou académique. Les systèmes et théories ne m'intéressent que pour autant qu'ils m'aident à saisir en tant qu'historien de la culture la formation, le fonctionnement et les formes d'attribution de sens de sociétés données. Je m'occupe plus particulièrement de leur impact sur la vie des acteurs de l'histoire, si possible au sens le plus personnalisé ou individuel du terme d'acteur, actant ou agissant. Ce qui me passionne dans l'histoire est l'interaction continue mais toujours changeante entre les personnes humaines ou des groupes bien cernés, leurs façons d'agir, les codes de conduite et autres outils qu'ils utilisent pour construire leur vie individuellement ou en commun, les interprétations symboliques de la réalité, et les transformations sociales et culturelles qui en découlent.

2 Mon attachement au français en tant qu'objet d'étude est en quelque sorte le fruit d'un hasard personnel, lié à la prolongation imprévue d'un séjour d'études en France qui aurait dû être limité à ma formation universitaire, et à mon insertion dans la société française par le biais familial. Par ailleurs, je suis connu comme un spécialiste de la période moderne - un terme malencontreux, très marqué par une conception linéaire de l'histoire, pour une époque qui était fertile en initiatives sociales, évolutions politiques et transformations culturelles autant en France que dans mon pays natal, les Pays-Bas actuels, mais qui n'a plus rien de « moderne » dans l'acception actuelle. L'intérêt que j'y porte est le fruit d'une autre série de hasards, liés à la rencontre de maîtres qui m'ont stimulé dans le sens de leurs propres passions. Mais il provient aussi de ma découverte 
personnelle d'un terrain historique où ont été jetés les fondements de l'Europe d'aujourd'hui, profondément divisée par ses stratégies identitaires tout en restant unie par une culture commune de base, dont les paramètres sont plus clairs que jamais, maintenant que l'Europe accueille des populations représentant une multitude d'expressions culturelles divergentes, voire adverses, venues de toutes parts. D'ailleurs, d'autres hasards de recherches m'ont depuis lors tourné vers l'Amérique coloniale.

3 Le choix de la période moderne n'a eu pour moi rien de prédestiné et n'a toujours rien de contraignant. Ainsi, le mémoire de maîtrise par lequel j'avais terminé mes études de théologie, préalablement à celles d'histoire, était axé sur le Moyen Âge, et avant de me concentrer sur le cœur de la période moderne, le XVII ${ }^{e}$ siècle batave et français, j'ai longuement flirté avec le XVIII siècle, puis la période révolutionnaire et ses suites, un amour qui laisse toujours des traces. J'y retrouve d'ailleurs à chaque détour la francophonie ou les Français eux-mêmes dans une multitude de thèmes: le français langue des rapports internationaux, le voyage de France et la pérégrination académique, la culture de cour conquérante, la mode et les amours, les livres et périodiques, les idéaux et pratiques révolutionnaires, la domination et l'oppression linguistique et culturelle, puis les grands actes d'institution du monde contemporain par la politique, l'éducation, la planification, la morale bourgeoise, une culture expansive et colonisatrice aussi bien dans les colonies au sens propre que dans les pays sœurs de l'Occident, etc. Tout cela proclame haut et fort que l'on ne saurait penser ou analyser la période moderne sans tenir compte de la place centrale de la France, des francophones de France et d'ailleurs, et du français.

4 Le regard que je jette sur l'histoire de l'enseignement du français langue étrangère ou seconde est donc un regard d'historien, le regard de celui qui scrute les occurrences et occasions saisies par les hommes et femmes, en tant que personnes dotées de la faculté du langage et praticiens de langues différemment constituées, pour se construire un réseau de contacts et de compréhension mutuels, achever la réalisation de projets, idéaux ou rêves collectifs, élargir leur horizon ou au contraire restreindre l'accès à leur univers. Soulignons l'importance de ce dernier élément : les restrictions, négations ou refus. En effet, chaque scientifique, érudit ou professionnel court toujours le risque de ne relever que le positif, les réussites de l'histoire et les actes documentés. Inconsciemment, l'interprétation se limite le plus souvent à ne mettre en avant que ce qui confirme ou exalte l'objet étudié, et à en accentuer les performances et exploits, tout en oubliant les facteurs d'échec, les occasions ratées et les refus, ou encore en négligeant les succès des concurrents qui utilement peuvent relativiser le récit de la conquête. Ainsi, le français de l'époque moderne demeure-t-il toujours en concurrence, parfois ardue, avec un triple faisceau linguistique : les langues anciennes, en particulier le latin, qui demeurent les langues de l'érudition internationale et universitaire; les langues régionales et les dialectes locaux, qui demeurent les langues d'intimité et de la vie communautaire du grand nombre ; et les langues étrangères qui continuent de régir le commerce des hommes et des biens dans de nombreux domaines et d'immenses régions du monde.

5 Je ne me considère donc pas un historien de plein droit du français langue étrangère ou seconde et de son enseignement, mais plutôt un historien de la culture et de l'éducation qui à l'occasion regarde le monde par le prisme de la pratique du français dans ces deux domaines, en reconnaissant que la place du français dans les sociétés européennes d'autrefois mérite bien un traitement privilégié mais certainement pas unique. La 
nuance est de taille, car elle implique un point de départ qui considère la langue comme un instrument neutre sur le terrain de la négociation culturelle et demeure toujours prêt à en souligner les limites et échecs au même titre que les réussites et les gloires.

6 La tâche primaire de l'historien est de construire son thème comme un objet historique, en accord avec un discours de la méthode qui vaille dans sa profession. Puis de présenter sur cet objet un discours interprétatif - discours qui peut être de facture littéraire, scientifique ou autre, et même de facture visuelle si la thématique s'y prête. Les méthodes historiques sont loin d'être uniques ou même univoques, mais dans tous les cas elles privilégient l'axe du temps et le domaine de l'agir humain, et partant les facteurs sociaux et culturels, même lorsqu'il s'agit d'analyser des structures, des systèmes ou des concepts théoriques. Dans le domaine qui nous concerne ici, c'est-àdire l'enseignement $d u$ français langue étrangère ou seconde, l'objet se situe à l'intersection de plusieurs champs historiques : l'éducation dans tous les sens du terme, y compris l'enseignement institutionnalisé (école, instituts, internats, cours régulièrement établis), formel (maîtres de langue, précepteurs) ou informel (guides choisis, l'autodidaxie par manuels ou sur le tas, l'immersion linguistique, etc.) ; la langue, formelle et standardisée, mais aussi ses variantes dialectales ou autres; le français, dans toute son extension sociale et culturelle et toute sa diffusion géographique, sans cette limitation quasi-automatique à la France dès que l'on en foule le sol ; enfin le rapport à l'étranger, qui introduit un facteur politique lié à la nation ou à la communauté sociolinguistique formalisée.

7 Dans le passé, l'accent primaire de la recherche tombait le plus souvent sur (l'enseignement de) la langue comme objet linguistique autonome correspondant aux règles internes de sa formation et de sa circulation (linguistique, didactique, lexicographique, etc.), ou comme véhicule littéraire ou intellectuel répondant aux règles normatives externes qui régissent sa forme, sa qualité (ou pureté), les règles de son usage, sa capacité d'expression, etc. L'historien, en revanche, se pose d'autres types de questions. Son objet est la langue en contexte, c'est-à-dire la langue comme objet d'action, d'intervention et de négociation dans le champ social des biens et valeurs culturels. Son discours n'est pas - ou seulement accessoirement - centré sur la langue elle-même mais sur ses usages, ses interactions dans les champs plurilingues, ses valeurs communautaires, son impact dans d'autres domaines ou secteurs de la communauté ou de la nation. Il s'intéresse avant tout au domaine social qui constitue la langue : sa pratique (sans pratiquants, pas de langue qui vaille ce mot), sa diffusion, son impact; et à son rôle dans le domaine culturel plus large, impliquant les valeurs, les symbolismes et les jugements de qualité. Bien au-delà de sa valeur d'usage dans la vie quotidienne, la langue est un objet de pouvoir interhumain, un instrument de domination interculturelle, et à l'occasion un moyen efficace pour obtenir un pouvoir alternatif, que ce soit dans l'intimité ou dans les rapports sociaux. Bien sûr, l'opposition entre l'objet et son usage est assez factice, mais elle permettra de distinguer de façon analytique entre deux approches scientifiques assez différentes du fait linguistique qui souvent ne se rencontrent guère, si elles ne s'ignorent pas carrément.

Le concept clé de cette deuxième approche peut être défini comme le marché linguistique - terme pris ici, bien entendu, dans le sens figuré du mot " marché ", quand bien même l'on découvre dans le commerce international des langues parfois des transactions qui renvoient assez clairement à son sens littéral. Dans cette activité "économique " l'enseignement de la langue joue un rôle formateur, préparatoire ou correcteur. Le 
marché linguistique est essentiellement un marché social et culturel, qui organise et définit les valeurs et pratiques d'usage dans les territoires politiques (nationaux, régionaux, communautaires, etc.), sociaux et culturels; il règle la concurrence, définit les domaines d'application des différentes langues qui se présentent, oriente les usagers vers des langues, dialectes ou sociolectes mieux adaptés aux usages pratiques et aux valeurs culturelles, au mouvement social ou aux conflits et intérêts collectifs, et fixe la métalangue commune du moment (la lingua franca). Comme l'histoire nous l'apprend, celle-ci, qui dans l'Europe moderne fut pendant un long moment le français, est loin d'être fixe ou universelle, car elle demeure sujette au jeu changeant des forces politiques et aux valeurs culturelles toujours en transformation. L'histoire d'une langue n'est jamais une histoire close, achevée, et on ne saurait postuler pour elle une évolution linéaire dans l'avenir proche ou lointain.

9 Nous pouvons considérer l'enseignement lui-même de la langue comme un bien culturel, objet possible d'une approche économique - le terme « économie » étant pris ici, tout comme la notion de marché, dans son acception la plus large, dépassant le purement mercantile pour englober toute forme d'échange réel, symbolique ou virtuel. Pour l'analyser et l'étudier dans cette perspective, on peut donc utilement avoir recours aux concepts et méthodes qui peuvent être empruntés au vocabulaire socioculturel de base de l'étude du marché, soit :

10 - l'offre : c'est-à-dire la langue en tant qu'objet constitué, la communauté linguistique qui la porte et transmet, les maîtres et les écoles ;

11 - la demande : les positions sociales, économiques, culturelles, politiques ou religieuses impliquant son usage ;

12 - les voies de transmission ou de transfert: les occasions de contact, les domaines d'application (l'économie et la vie quotidienne, bien sûr, mais aussi, et plus particulièrement des domaines spécifiques où la langue reflète un savoir de groupe surdimensionné, tels que la gestion du corps, la mode, la cuisine, le savoir-vivre, la science, la piété ou la philosophie) ;

13 - l'appropriation de la langue, de son enseignement et de ses formes, modalités et occasions d'usage par la communauté, la nation, des groupes particuliers, la famille, voire la personne particulière dans son expression intime.

14 C'est par une analyse combinatoire intelligente de tels facteurs que l'on pourra pleinement rendre compte des variations de diffusion et d'intensité, de quantité et de qualité de l'usage du français langue étrangère ou seconde, et du rôle que son enseignement a pu jouer pour les réaliser. Même limité à l'Europe moderne, ce genre d'analyse pourra aider à dégager des modèles utiles pour la gestion de la langue sur le marché linguistique d'aujourd'hui, sans oublier ses rôles possibles dans l'avenir. 


\section{AUTEUR}

WILLEM FRIJHOFF

Université Libre, Amsterdam / Université Érasme, Rotterdam 